\title{
Domination in Controlled and Observed Distributed Parameter Systems
}

\author{
L. Afifi ${ }^{1}$, M. Joundi ${ }^{1}$, E. M. Magri ${ }^{1}$, A. El Jai ${ }^{2}$ \\ ${ }^{1}$ Department of Mathematics and Computer Science, Faculty of Sciences Ain Chock, \\ University of Hassan II Casablanca, Casablanca, Morocco \\ ${ }^{2}$ MEPS-Systems Theory, University of Perpignan, Perpignan, France \\ Email: larbi_afifi@yahoo.fr, 1.afifi@fsac.ac.ma, meriemjoundi@yahoo.fr,magrielmostafa@hotmail.com, aej@univ-perp.fr
}

Received November 16, 2012; revised December 23, 2012; accepted January 7, 2013

Copyright (C) 2013 L. Afifi et al. This is an open access article distributed under the Creative Commons Attribution License, which permits unrestricted use, distribution, and reproduction in any medium, provided the original work is properly cited.

\begin{abstract}
We consider and we study a general concept of domination for controlled and observed distributed systems. We give characterization results and the main properties of this notion for controlled systems, with respect to an output operator. We also examine the case of actuators and sensors. Various other situations are considered and applications are given. Then, we extend this study by comparing observed systems with respect to a control operator. Finally, we study the relationship between the notion of domination and the compensation one, in the exact and weak cases.
\end{abstract}

Keywords: Distributed Systems; Domination; Actuators; Sensors; Compensation

\section{Introduction}

This work concerns the systems analysis and more precisely a general concept of domination. This notion consists to study the possibility of comparison or classification of systems. It was introduced firstly in [1] for controlled and observed lumped systems and then in [2] for a class of distributed parameter systems. The developed approach concerns separately the input and output operators. Various results are given and illustrated by applications and examples. A duality between the two cases is established. An extension of [2] to the regional case is given in [3]. The regional aspect of this problem is motivated by the fact that a system may dominates another one in a region $\omega$, but not on the whole geometrical support $\Omega$ of the system.

Let us note that in the case of the dual notions of observability and controllability, the literature is very rich. However, the purpose is different and generally, the main problem is how to reconstruct the state of the considered system or to reach a desired state, i.e. to study if a system is (or not) observable or controllable.

In this paper, we consider and we study a more general domination problem in the case of a class of controlled and observed systems [4-6]. The developed approach depends on the different parameters of the considered systems, such their dynamics, their input and output operators. Indeed, we consider without loss of generality, a class of linear distributed systems as follows

$$
\left\{\begin{array}{l}
\dot{z}(t)=A z(t)+B u(t) ; 0<t<T \\
z(0)=z_{0}
\end{array}\right.
$$

where $A$ generates a strongly continuous semi-group (s.c.s.g.) $(S(t))_{t \geq 0}$ on the state $Z . B \in \mathcal{L}(U, Z)$, $u \in L^{2}(0, T ; U) ; \quad Z$ and $U$ are respectively the state and the control spaces, assumed to be Hilbert spaces. The system (1) is augmented with the following output equation

$$
y(t)=C z(t)
$$

with $C \in \mathcal{L}(Z, Y), \quad Y$ is the observation space, a Hilbert space. The operator $A$ is the dynamics of the system, the operators $B$ and $C$ are respectively the input and output operators. The state $z$ of the system at time $t$ is given by

$$
z(t)=S(t) z_{0}+H_{t} u
$$

where

$$
H_{t} u=\int_{0}^{t} S(t-s) B u(s) \mathrm{d} s
$$

and the observation by

$$
y(t)=C S(t) z_{0}+C_{t} u
$$

The first problem consists to study a possible com- 
parison of controlled systems as system (1), with respect to an output operator $C$. We give the main properties and characterization results. The case of sensors and actuators is also examined. Illustrative examples and applications are presented and various other situations are examined.

Then, an analogous study concerning the domination of observed systems, with respect to an input operator $B$, is given. Finally, we study the relationship between the notion of domination and the compensation problem $[7,8]$.

\section{Domination for Controlled Systems}

\subsection{Problem Statement and Definitions}

We consider the following linear distributed systems

$$
\begin{aligned}
& \left(S_{1}\right)\left\{\begin{array}{l}
\dot{z}_{1}(t)=A_{1} z_{1}(t)+B_{1} u_{1}(t) ; 0<t<T \\
z_{1}(0)=z_{1,0} \in Z
\end{array}\right. \\
& \left(S_{2}\right)\left\{\begin{array}{l}
\dot{z}_{2}(t)=A_{2} z_{2}(t)+B_{2} u_{2}(t) ; 0<t<T \\
z_{2}(0)=z_{2,0} \in Z
\end{array}\right.
\end{aligned}
$$

where, for $i=1,2 ; A_{i}$ is a linear operator generating a s.c.s.g. $\left(S_{i}(t)\right)_{t \geq 0}$ on the state space $Z . \quad B_{i} \in \mathcal{L}\left(U_{i}, Z\right)$, $u_{i} \in L^{2}\left(0, T ; U_{i}\right) ; U_{i}$ is a control space. The systems $\left(S_{1}\right)$ and $\left(S_{2}\right)$ are respectively augmented with the output equations

$$
\left(E_{i}\right) y_{i}(t)=C z_{i}(t) \text { for } i=1,2 ; C \in \mathcal{L}(Z, Y)
$$

The state of $\left(S_{i}\right)$ at the final time $T$ is given by

$$
z_{i}(T)=S_{i}(T) z_{i, 0}+H_{i} u_{i}
$$

where

$$
\begin{aligned}
& H_{i}: L^{2}\left(0, T ; U_{i}\right) \rightarrow Z \\
& u_{i} \rightarrow \int_{0}^{T} S_{i}(T-s) B_{i} u_{i}(s) \mathrm{d} s
\end{aligned}
$$

The corresponding observation at time $T$ is given by

$$
y_{i}(T)=C S_{i}(T) z_{i, 0}+C H_{i} u_{i}
$$

The purpose is to study a possible comparison of systems $\left(S_{1}\right)$ and $\left(S_{2}\right)$ (or the input operators $B_{1}$ and $B_{2}$ if $\left.A_{1}=A_{2}\right)$ with respect to the output operator $C$.

It is based on the dynamics $A_{1}$ and $A_{2}$, the control operators $B_{1}, B_{2}$ and the observation operator $C$. Without loss of generality, one can assume that $Z_{1,0}=$ $z_{2,0} \equiv 0$. We introduce hereafter the corresponding notion of domination.

Definition 1. We say that

1) $\left(S_{1}\right)$ dominates $\left(S_{2}\right)$ (or the pair $\left(A_{1}, B_{1}\right)$ dominates $\left.\left(A_{2}, B_{2}\right)\right)$ exactly on $[0, T]$, with respect to the operator $C$, if

$$
\operatorname{Im}\left(\mathrm{CH}_{2}\right) \subset \operatorname{Im}\left(\mathrm{CH}_{1}\right)
$$

2) $\left(S_{1}\right)$ dominates $\left(S_{2}\right)$ (or the pair $\left(A_{1}, B_{1}\right)$ dominates $\left.\left(A_{2}, B_{2}\right)\right)$ weakly on $[0, T]$, with respect to the operator $C$, if

$$
\overline{\operatorname{Im}\left(\mathrm{CH}_{2}\right)} \subset \overline{\operatorname{Im}\left(\mathrm{CH}_{1}\right)}
$$

In this situation, we note respectively

$$
\begin{aligned}
& \left(A_{2}, B_{2}\right) \underset{C}{\leq}\left(A_{1}, B_{1}\right) \\
& \text { and }\left(A_{2}, B_{2}\right) \underset{C}{\underset{C}{\lesssim}}\left(A_{1}, B_{1}\right)
\end{aligned}
$$

Let us give following properties and remarks :

1) Obviously, the exact domination with respect to an output operator $C$, implies the weak one with respect to $C$. The converse is not true, this is shown in [2] for $A_{1}=A_{2}$ and $C=I$ ).

2) If the system $\left(S_{1}\right)$ is controllable exactly (respectively weakly), or equivalently

$$
\operatorname{Im}\left(H_{1}\right)=Z \quad\left(\text { respectively } \overline{\operatorname{Im}\left(H_{1}\right)}=Z\right)
$$

then $\left(S_{1}\right)$ dominates exactly (respectively weakly) any system $\left(S_{2}\right)$, with respect to any output operator $C$.

3 ) In the case where $A_{1}=A_{2},\left(S_{1}\right)$ dominates $\left(S_{2}\right)$ exactly (respectively weakly), we say simply that $B_{1}$ dominates $B_{2}$ exactly (respectively weakly). Then, we note

$$
B_{2} \leq B_{1}\left(\text { respectively } B_{2} \underset{C}{\lesssim} B_{1}\right) .
$$

Hence, one can consider a single system with two inputs as follows

$$
\text { (S) }\left\{\begin{array}{l}
\dot{z}(t)=A z(t)+B_{1} u_{1}(t)+B_{2} u_{2}(t) ; 0<t<T \\
z(0)=z_{0} \in Z
\end{array}\right.
$$

augmented with an output equation

$$
(E) y(t)=C z(t)
$$

In this case, the domination of control operators $B_{1}$ and $B_{2}$, with respect to the observation operator $C$, is similar. The definitions and results remain practically the same.

4) The exact or weak domination of systems (or operators) is a transitive and reflexive relation, but it is not antisymmetric. Thus, for example in the case where $A_{1}=A_{2}$, for any non-zero operator $B_{1} \neq 0$ and $\alpha \neq 0$, we have $\operatorname{Im}\left(C H_{1}\left(B_{1}\right)\right)=\operatorname{Im}\left(C H_{1}\left(\alpha B_{1}\right)\right)$, even if $B_{1} \neq \alpha B_{1}$ for $\alpha \neq 1$.

5) Concerning the relationship with the notion of remediability $[7,8]$, we consider without loss of generality, a class of linear distributed systems described by the following state equation

${ }^{1} H_{1}\left(B_{1}\right)$ denotes the operator $H_{1}$ corresponding to $B_{1}$, i.e. defined by $H_{1}\left(B_{1}\right) u_{1}=\int_{0}^{T} S_{1}(T-s) B_{1} u_{1}(s) \mathrm{d} s$. 


$$
\left\{\begin{array}{l}
\dot{z}(t)=A z(t)+B u(t)+d(t) ; 0<t<T \\
z(0)=z_{0}
\end{array}\right.
$$

where $d \in L^{2}(0, T ; Z)$ is a known or unknown disturbance. The system (12) is augmented with the following output equation

$$
y(t)=C z(t)
$$

The state $z$ of the system at time $T$ is given by

$$
z(T)=S(T) z_{0}+H u+R d
$$

where

$$
R d=\int_{0}^{T} S(T-s) d(s) \mathrm{d} s
$$

If the system (12), augmented with (13), is exactly (respectively weakly) remediable on $[0, T]$, or equivalently $\operatorname{Im}(C R) \subset \operatorname{Im}(C H) \quad$ (respectively $\overline{\operatorname{Im}(C R)} \subset$ $\overline{\operatorname{Im}(C H)})$, then $B_{1}$ dominates any operator $B_{2}$ exactly (respectively weakly) with respect to the operator C.

6) For $C=I$ and $A_{1}=A_{2}$, one retrieve the particular notion of domination as in [2].

We give hereafter characterization results concerning the exact and weak domination.

\subsection{Characterizations}

The following result gives a characterization of the exact domination with respect to the output operator $C$.

Proposition 2. The following properties are equivalent

1) The system $\left(S_{1}\right)$ dominates exactly $\left(S_{2}\right)$ with respect to the operator $C$.

2) For any $u_{2} \in L^{2}\left(0, T ; U_{2}\right)$, there exists $u_{1} \in$ $L^{2}\left(0, T ; U_{1}\right)$ such that

$$
\mathrm{CH}_{1} u_{1}+\mathrm{CH}_{2} \mathrm{u}_{2}=0
$$

3) There exists $\gamma>0$ such that for any $\theta \in Y^{\prime}$, we have

$$
\begin{cases}\left.\frac{\partial z(x, t)}{\partial t}=\alpha \frac{\partial^{2} z(x, t)}{\partial x^{2}}+\beta z(x, t)+g(x) u(t) \text { in }\right] 0,1[\times] 0, T[ \\ z(0, t)=z(1, t)=0 & \text { in }] 0, T[ \\ z(x, 0)=z_{0}(x) & \text { in }] 0,1[\end{cases}
$$

The operator $M(\alpha, \beta)=\alpha \frac{\partial^{2}}{\partial x^{2}}+\beta I \quad$ generates the s.c.s.g. $\left(S_{(\alpha, \beta)}(t)\right)_{t \geq 0}$ defined by

$$
S_{(\alpha, \beta)}(t) z=\sum_{n=1}^{+\infty} \mathrm{e}^{\left(\beta-\alpha n^{2} \pi^{2}\right) t} \sum_{j=1}^{r_{n}}\left\langle z, \varphi_{n j}\right\rangle \varphi_{n j}
$$

where $\left(\varphi_{n}\right)_{n}$, with $\varphi_{n}(x)=\sqrt{2} \sin (n \pi x)$, is a complete

$$
\left\|B_{2}^{*} S_{2}^{*}(T-.) C^{*} \theta\right\|_{L^{2}\left(0, T: U_{2}\right)} \leq \gamma\left\|B_{1}^{*} S_{1}^{*}(T-.) C^{*} \theta\right\|_{L^{2}\left(0, T: U_{1}\right)}
$$

\section{Proof.}

The equivalence between i) and ii) derives from the definition.

The equivalence between ii) and iii) is a consequence of the fact that if $X, Y$ and $Z$ are Banach spaces; $P \in \mathcal{L}(X, Z)$ and $Q \in \mathcal{L}(Y, Z)$, then

$$
\operatorname{Im}(P) \subset \operatorname{Im}(Q)
$$

if and only if, there exists $\gamma>0$ such that for any $z^{*} \in Z^{\prime}$, we have

$$
\left\|P^{*} z^{*}\right\|_{X^{\prime}} \leq \gamma\left\|Q^{*} z^{*}\right\|_{Y^{\prime}}
$$

where $X^{\prime}, Y^{\prime}$ and $Z^{\prime}$ are respectively the dual spaces of $X, Y$ and $Z$.

Concerning the weak case, we have the following characterization result.

\section{Proposition 3.}

The system $\left(S_{1}\right)$ dominates $\left(S_{2}\right)$ weakly, with respect to $C$, if and only if

$$
\operatorname{ker}\left[B_{1}^{*} S_{1}^{*}(.) C^{*}\right] \subset \operatorname{ker}\left[B_{2}^{*} S_{2}^{*}(.) C^{*}\right]
$$

Proof.

Derives from the definition and the fact that $\overline{\operatorname{Im}\left(\mathrm{CH}_{2}\right)} \subset \overline{\operatorname{Im}\left(\mathrm{CH}_{1}\right)}$ is equivalent to $\operatorname{ker}\left(\mathrm{CH}_{1}\right)^{*}$ $\subset \operatorname{ker}\left(\mathrm{CH}_{2}\right)^{*}$.

It is well known that the choice of the input operator play an important role in the controllability of a system [4-6,9-11]. Here also, the domination for controlled systems, with respect to an output operator $C$, depends on the dynamics $A_{i}$ and particularly on the choice of the control operators $B_{i}$. However, even if $B_{1}=B_{2}=B$ (with the same actuator), the pair $\left(A_{1}, B\right)$ may dominates $\left(A_{2}, B\right)$. This is illustrated in the the following example.

Example 4. We consider the system described by the one dimension equation

system of eigenfunctions of $M(\alpha, \beta)$ associated to the eigenvalues $\lambda_{n}=\gamma-\beta n^{2} \pi^{2} \quad(\alpha, \beta \in \mathbb{R})$.

For $z^{*} \in Z^{\prime 2}(0,1)$, we have

$$
\begin{aligned}
& \left\|B^{*} S_{(\alpha, \beta)}^{*}(t) z^{*}\right\|_{L^{2}(0, T ; \mathbb{R})}^{2} \\
& =\sum_{n=1}^{+\infty} \int_{0}^{T} \mathrm{e}^{2\left(\beta-\alpha n^{2} \pi^{2}\right) t} \sum_{j=1}^{r_{n}}\left\langle z, \varphi_{n j}\right\rangle^{2}\left\langle g, \varphi_{n j}\right\rangle^{2} \mathrm{~d} t
\end{aligned}
$$


Hence, if $g=\varphi_{n_{0}} \quad\left(n_{0} \geq 1\right)$, Equation (17) becomes

$\left\|B^{*} S_{(\alpha, \beta)}^{*}(t) z^{*}\right\|_{L^{2}(0, T ; \mathbb{R})}^{2}=\sum_{n=1}^{+\infty} \int_{0}^{T} \mathrm{e}^{2\left(\beta-\alpha n^{2} \pi^{2}\right) t}\left\langle z, \varphi_{n_{0}}\right\rangle^{2} \mathrm{~d} t$

Let $A_{1}=M_{1,0}=\frac{\partial^{2}}{\partial x^{2}} \quad$ and $\quad A_{2}=M_{1, \beta}=\frac{\partial^{2}}{\partial x^{2}}+\beta I$; $\beta \neq 0$.

The corresponding semi-groups, noted $\left(S_{1}(t)\right)_{t \geq 0}$ and $\left(S_{2}(t)\right)_{t \geq 0}$, are respectively defined by

$$
S_{1}(t) z=\sum_{n=1}^{+\infty} \mathrm{e}^{-n^{2} \pi^{2} t} \sum_{j=1}^{r_{n}}\left\langle z, \varphi_{n j}\right\rangle \varphi_{n j}
$$

and

$$
S_{2}(t) z=\sum_{n=1}^{+\infty} \mathrm{e}^{\left(\beta-n^{2} \pi^{2}\right) t} \sum_{j=1}^{r_{n}}\left\langle z, \varphi_{n j}\right\rangle \varphi_{n j}
$$

Then for $B_{1}=B_{2}=B$, with $B u=\varphi_{n_{0}} u$

1) If $\beta>0$, then for any $z^{*} \in Z^{\prime}$, we have

$$
\begin{aligned}
\left\|B^{*} S_{1}^{*}(t) z^{*}\right\|_{L^{2}(0, T ; \mathbb{R})}^{2} & =\sum_{n=1}^{+\infty} \int_{0}^{T} \mathrm{e}^{-2 n^{2} \pi^{2} t}\left\langle z^{*}, \varphi_{n}\right\rangle^{2} \mathrm{~d} t \\
& \leq \sum_{n=1}^{+\infty} \int_{0}^{T} \mathrm{e}^{2\left(\beta-n^{2} \pi^{2}\right) t}\left\langle z^{*}, \varphi_{n}\right\rangle^{2} \mathrm{~d} t \\
& =\left\|B^{*} S_{2}^{*}(t) z^{*}\right\|_{L^{2}(0, T ; \mathbb{R})}^{2}
\end{aligned}
$$

consequently, the pair $\left(A_{2}, B\right)$ dominates the pair $\left(A_{1}, B\right)$ exactly, and hence weakly.

2) If $\beta<0$, then for any $z^{*} \in Z^{\prime}$,

$$
\begin{aligned}
\left\|B^{*} S_{2}^{*}(t) z^{*}\right\|_{L^{2}(0, T ; \mathbb{R})}^{2} & =\sum_{n=1}^{+\infty} \int_{0}^{T} \mathrm{e}^{2\left(\beta-n^{2} \pi^{2}\right) t}\left\langle z^{*}, \varphi_{n}\right\rangle^{2} \mathrm{~d} t \\
& \leq \sum_{n=1}^{+\infty} \int_{0}^{T} \mathrm{e}^{-2 n^{2} \pi^{2} t}\left\langle z^{*}, \varphi_{n}\right\rangle^{2} \mathrm{~d} t \\
& =\left\|B^{*} S_{1}^{*}(t) z^{*}\right\|_{L^{2}(0, T ; \mathbb{R})}^{2}
\end{aligned}
$$

Hence, the pair $\left(A_{1}, B\right)$ dominates the pair $\left(A_{2}, B\right)$ exactly (and weakly).

In the next section, we examine the case of a finite number of actuators, and then the case where the observation is given by sensors.

\subsection{Case of Actuators and Sensors}

This section is focused on the notions of actuators and sensors $[4,8,10]$, i.e. on input and output operators. In what follows, we assume that $Z=L^{2}(\Omega)$ and, without loss of generality, we consider the analytic case where $A_{1}$ and $A_{2}$ generate respectively the s.c.s.g. $\left(S_{1}(t)\right)_{t \geq 0}$ and $\left(S_{2}(t)\right)_{t \geq 0}$ defined by

$$
S_{1}(t) z=\sum_{n=1}^{+\infty} \mathrm{e}^{\lambda_{n} t} \sum_{j=1}^{r_{n}}\left\langle z, \varphi_{n j}\right\rangle \varphi_{n j}
$$

and

$$
S_{2}(t) z=\sum_{n=1}^{+\infty} \mathrm{e}^{\gamma_{n} t} \sum_{j=1}^{s_{n}}\left\langle z, \psi_{n j}\right\rangle \psi_{n j}
$$

where $\left\{\varphi_{n j}, j=1, \cdots, r_{n} ; n \geq 1\right\}$ is a complete orthonormal basis of eigenfunctions of $A_{1}$, associated to the real eigenvalues $\left(\lambda_{n}\right)_{n \geq 1}$ such that $\lambda_{1}>\lambda_{2}>\lambda_{3}>\cdots ; r_{n}$ is the multiplicity of $\lambda_{n}$.

$\left\{\psi_{n j}, j=1, \cdots, s_{n} ; n \geq 1\right\}$ is a complete orthonormal basis of eigenfunctions of $A_{2}$, associated to the real eigenvalues $\left(\gamma_{n}\right)_{n \geq 1}$ such that $\gamma_{1}>\gamma_{2}>\gamma_{3}>\cdots ; s_{n}$ is the multiplicity of $\gamma_{n}$.

\subsubsection{Case of Actuators}

In the case where $\left(S_{1}\right)$ is excited by $p$ zone actuators $\left(\Omega_{i}, g_{i}\right)_{1 \leq i \leq p}$, we have $U_{1}=I R^{p}$ and

$$
B_{1} u(t)=\sum_{i=1}^{p} g_{i} u_{i}(t)
$$

where $u=\left(u_{1}, \cdots, u_{p}\right)^{t r} \in L^{2}\left(0, T ; I R^{p}\right)$ and $g_{i} \in L^{2}(\Omega)$; $\Omega_{i}=\operatorname{supp}\left(g_{i}\right) \subset \Omega$. We have

$$
B_{1}^{*} z=\left(\left\langle g_{1}, z\right\rangle, \cdots,\left\langle g_{p}, z\right\rangle\right)^{t r}
$$

By the same, if $\left(S_{2}\right)$ is excited by $q$ zone actuators $\left(D_{i}, h_{i}\right)_{1 \leq i \leq q}$, we have $U_{2}=I R^{q}$ and

$$
B_{2} v(t)=\sum_{i=1}^{q} h_{i} v_{i}(t)
$$

with $v=\left(v_{1}, \cdots, v_{q}\right)^{t r} \in L^{2}\left(0, T ; I R^{q}\right), h_{i} \in L^{2}(\Omega)$,

$D_{i}=\operatorname{supp}\left(h_{i}\right) \subset \Omega$ and

$$
B_{2}^{*} z=\left(\left\langle h_{1}, z\right\rangle, \cdots,\left\langle h_{q}, z\right\rangle\right)^{t r}
$$

As it will be seen in the next section, this leads to characterization results depending on $C$ and the corresponding controllability matrix, and then on the observability one in the case where the observation is given by a finite number of sensors. First, let us show the following preliminary result.

Proposition 5. We have

$$
\begin{aligned}
& \operatorname{ker}\left(B_{1}^{*} S_{1}^{*}(.) C^{*}\right) \\
& =\left\{\theta \in Y^{\prime} / \forall n \in \mathbb{N}^{*},\left(\left\langle C^{*} \theta, \varphi_{n j}\right\rangle\right)_{1 \leq j \leq r_{n}} \in \operatorname{ker}\left(M_{n}\right)\right\}
\end{aligned}
$$

and

$$
\begin{aligned}
& \operatorname{ker}\left(B_{2}^{*} S_{2}^{*}(.) C^{*}\right) \\
& =\left\{\theta \in Y^{\prime} / \forall n \in \mathbb{N}^{*},\left(\left\langle C^{*} \theta, \psi_{n j}\right\rangle\right)_{1 \leq j \leq s_{n}} \in \operatorname{ker}\left(Q_{n}\right)\right\}
\end{aligned}
$$

where $M_{n}$ and $Q_{n}$ are the corresponding controllability matrices defined by 


$$
M_{n}=\left(\left\langle g_{i}, \varphi_{n j}\right\rangle\right)_{1 \leq i \leq p ; 1 \leq j \leq r_{n}}
$$

and

$$
Q_{n}=\left(\left\langle h_{i}, \psi_{n j}\right\rangle\right)_{1 \leq i \leq q ; 1 \leq j \leq s_{n}}
$$

Proof. We have

$$
B_{1}^{*} S_{1}^{*}(t) C^{*} \theta=\left(\sum_{n=1}^{+\infty} \mathrm{e}^{\lambda_{n} t} \sum_{j=1}^{r_{n}}\left\langle C^{*} \theta, \varphi_{n j}\right\rangle\left\langle g_{i}, \varphi_{n j}\right\rangle\right)_{1 \leq i \leq p}
$$

Therefore, $\theta \in \operatorname{ker}\left(B_{1}^{*} S_{1}^{*}(.) C^{*}\right)$ if and only if $\sum_{n=1}^{+\infty} \mathrm{e}^{\lambda_{n} t} \sum_{j=1}^{r_{n}}\left\langle C^{*} \theta, \varphi_{n j}\right\rangle\left\langle g_{i}, \varphi_{n j}\right\rangle=0 ; \forall i \in\{1, \cdots, p\}, \forall t \geq 0$

By analyticity, this is equivalent to

$$
\sum_{j=1}^{r_{n}}\left\langle C^{*} \theta, \varphi_{n j}\right\rangle\left\langle g_{i}, \varphi_{n j}\right\rangle=0 ; \forall n \geq 1, \forall i \in\{1, \cdots, p\}
$$

or

$$
B_{1}^{*} S_{1}^{*}(t) C^{*} \theta=0 \Leftrightarrow v_{n}(\theta) \in \operatorname{ker}\left(M_{n}\right), \forall n \geq 1
$$

where

$$
v_{n}(\theta)=\left(\left\langle C^{*} \theta, \varphi_{n j}\right\rangle\right)_{j=1, r_{n}}
$$

The proof of the second equality of the proposition is similar.

The following result deriving from proposition 2, gives characterizations of exact and weak domination in the case of actuators.

Proposition 6.

1) $\left(S_{1}\right)$ dominates $\left(S_{2}\right)$ exactly with respect to the operator $C$, if and only if there exists $\gamma>0$ such that for any $\theta \in Y^{\prime}$, we have

$$
\begin{aligned}
& \left\|\left(\sum_{n=1}^{+\infty} \mathrm{e}^{\gamma_{n} t} \sum_{j=1}^{s_{n}}\left\langle C^{*} \theta, \psi_{n j}\right\rangle\left\langle h_{i}, \psi_{n j}\right\rangle\right)_{1 \leq i \leq q}\right\|_{L^{2}\left(0, T ; \mathbb{R}^{q}\right)} \\
& \leq \gamma\left\|\left(\sum_{n=1}^{+\infty} \mathrm{e}^{\lambda_{n} t} \sum_{j=1}^{r_{n}}\left\langle C^{*} \theta, \varphi_{n j}\right\rangle\left\langle g_{i}, \varphi_{n j}\right\rangle\right)_{1 \leq i \leq p}\right\|_{L^{2}\left(0, T ; \mathbb{R}^{p}\right)}
\end{aligned}
$$

2) $\left(S_{1}\right)$ dominates $\left(S_{2}\right)$ weakly with respect to the operator $C$, if and only if for any $\theta \in Y^{\prime}$, we have

$$
\begin{aligned}
& {\left[\forall n \in \mathbb{N}^{*},\left(\left\langle C^{*} \theta, \varphi_{n j}\right\rangle\right)_{1 \leq j \leq r_{n}} \in \operatorname{ker} M_{n}\right]} \\
& \Rightarrow\left[\forall n \in \mathbb{N}^{*},\left(\left\langle C^{*} \theta, \psi_{n j}\right\rangle\right)_{1 \leq j \leq s_{n}} \in \operatorname{ker} Q_{n}\right]
\end{aligned}
$$

Let us note that if $A_{1}=A_{2}$, the domination concerns the operators $B_{1}$ and $B_{2}$, and then the corresponding actuators. This leads to the following definition.

Definition 7. If $\left(S_{1}\right)$ dominates $\left(S_{2}\right)$ exactly (respectively weakly) with respect to the operator $C$, we say that $\left(\Omega_{i}, g_{i}\right)_{1 \leq i \leq p}$ dominate $\left(D_{i}, h_{i}\right)_{1 \leq i \leq q}$ exactly (respectively weakly) with respect to $C$.

In the usual case, the observation is given by sensors. This is examined in following section.

\subsubsection{Case of Sensors}

Now, if the output is given by $m$ sensors $\left(E_{i}, f_{i}\right)_{1 \leq i \leq m}$, we have

$$
C z=\left(\begin{array}{c}
\left\langle z, f_{1}\right\rangle \\
\vdots \\
\left\langle z, f_{m}\right\rangle
\end{array}\right) \in \mathbb{R}^{m}
$$

and

$$
C^{*} \theta=\sum_{i=1}^{m} \theta_{i} f_{i} \quad \text { for } \quad \theta \in \mathbb{R}^{m}
$$

We have the following proposition.

Proposition 8. $\left(S_{1}\right)$ dominates $\left(S_{2}\right)$ weakly with respect to the sensors $\left(E_{i}, f_{i}\right)_{1 \leq i \leq m}$, if and only if

$$
\bigcap_{n \geq 1} \operatorname{ker}\left(M_{n} G_{n}^{t r}\right) \subset \bigcap_{n \geq 1} \operatorname{ker}\left(Q_{n} R_{n}^{t r}\right)
$$

where $G_{n}$ and $R_{n}$ are the corresponding observability matrices defined by

$$
G_{n}=\left(\left\langle f_{i}, \varphi_{n j}\right\rangle\right)_{1 \leq i \leq m ; 1 \leq j \leq r_{n}}
$$

and

$$
R_{n}=\left(\left\langle f_{i}, \psi_{n j}\right\rangle\right)_{1 \leq i \leq m ; 1 \leq j \leq s_{n}}
$$

Proof. $\left(S_{1}\right)$ dominates $\left(S_{2}\right)$ weakly with respect to the sensors $\left(E_{i}, f_{i}\right)_{1<i<m}$, if and only if, for any $\theta=\left(\theta_{k}\right)_{1 \leq k \leq m} \in \mathbb{R}^{m}$,

$$
\forall n \in \mathbb{N}^{*},\left(\sum_{i=1}^{m} \theta_{k}\left\langle f_{k}, \varphi_{n j}\right\rangle\right)_{1 \leq j \leq r_{n}} \in \operatorname{ker}\left(M_{n}\right)
$$

implies that

$$
\forall n \in \mathbb{N}^{*},\left(\sum_{i=1}^{m} \theta_{k}\left\langle f_{k}, \psi_{n j}\right\rangle\right)_{1 \leq j \leq s_{n}} \in \operatorname{ker}\left(Q_{n}\right)
$$

or equivalently, for any $\theta \in \mathbb{R}^{m}$,

$$
\left[\forall n \geq 1, \theta \in \operatorname{ker}\left(M_{n} G_{n}^{*}\right)\right] \Rightarrow\left[\forall n \geq 1, \theta \in \operatorname{ker}\left(Q_{n} G_{n}^{*}\right)\right]
$$

we then have the result.

Let us give the following remarks.

1) If $A_{1}=A_{2}$, we have $G_{n}=R_{n}$, for $n \geq 1$.

2) One actuator may dominates $p$ actuators $(p>1)$, with respect to an output operator $C$ (sensors).

3) In the case of one actuator and one sensor, i.e. for $p=q=1$ and $m=1$, we have

$$
\begin{aligned}
& M_{n}=\left(\left\langle g, \varphi_{n 1}\right\rangle, \cdots,\left\langle g, \varphi_{n r_{n}}\right\rangle\right), \\
& Q_{n}=\left(\left\langle h, \psi_{n 1}\right\rangle, \cdots,\left\langle h, \psi_{n s_{n}}\right\rangle\right)
\end{aligned}
$$


and

$$
G_{n}^{t r}=\left(\begin{array}{c}
\left\langle f, \varphi_{n 1}\right\rangle \\
\vdots \\
\left\langle f, \varphi_{n r_{n}}\right\rangle
\end{array}\right), R_{n}^{t r}=\left(\begin{array}{c}
\left\langle f, \psi_{n 1}\right\rangle \\
\vdots \\
\left\langle f, \psi_{n s_{n}}\right\rangle
\end{array}\right)
$$

Then

$$
\begin{aligned}
& M_{n} G_{n}^{t r}=\left(\sum_{j=1}^{r_{n}}\left\langle g, \varphi_{n j}\right\rangle\left\langle f, \varphi_{n j}\right\rangle\right) \\
& Q_{n} R_{n}^{t r}=\left(\sum_{j=1}^{s_{n}}\left\langle h, \psi_{n j}\right\rangle\left\langle f, \psi_{n j}\right\rangle\right)
\end{aligned}
$$

4) In the case of a finite number of sensors, the exact and weak domination are equivalent.

\section{Application to Diffusion Systems}

To illustrate previous results and other specific situations, we consider without loss of generality, a class of diffusion systems described by the following parabolic equa- tion.

$$
\left\{\begin{array}{l}
\left.\frac{\partial z(x, t)}{\partial t}=\Delta z(x, t)+g(x) u(t) \quad \Omega \times\right] 0, T[ \\
z(x, 0)=0 \quad \Omega \\
z(\xi, t)=0 \quad \partial \Omega \times] 0, T[
\end{array}\right.
$$

where $\Omega$ is a bounded subset of $R^{n}$ with a sufficiently regular boundary $\partial \Omega=\Gamma ; Z=L^{2}(\Omega)$ and $A Z=\Delta Z$ for $z \in D(A)=H^{2}(\Omega) \cap H_{0}^{1}(\Omega)$. (S) is augmented with the output equation

$$
\text { (E) } y(t)=C z(t), 0<t<T
$$

We examine respectively, hereafter the case of one and two space dimension.

\subsection{One Dimension Case}

In this section, we consider the systems $\left(S_{1}\right)$ and $\left(S_{2}\right)$ described by the following one dimension equations, with $\Omega=] 0, a\left[\right.$ and $A_{1}=A_{2}=\Delta$.

$$
\begin{aligned}
& \left(S_{1}\right) \begin{cases}\frac{\partial z_{1}(x, t)}{\partial t}=\frac{\partial^{2} z_{1}(x, t)}{\partial x^{2}}+g(x) u_{1}(t) & \text { in }] 0, a[\times] 0, T[ \\
z_{1}(0, t)=z_{1}(a, t)=0 & \text { in }] 0, T[ \\
z_{1}(x, 0)=0 & \text { in }] 0, a[\end{cases} \\
& \left(S_{2}\right)\left\{\begin{array}{lr}
\left.\frac{\partial z_{2}(x, t)}{\partial t}=\frac{\partial^{2} z_{2}(x, t)}{\partial x^{2}}+h(x) u_{2}(t) \text { in }\right] 0, a[\times] 0, T[ \\
z_{2}(0, t)=z_{2}(a, t)=0 & \text { in }] 0, T[ \\
z_{2}(x, 0)=0 & \text { in }] 0, a[
\end{array}\right.
\end{aligned}
$$

$A=\Delta$ admits a complete orthonormal system of eigenfunctions $\left(\varphi_{n}\right)_{n \in \mathbb{N}^{*}}$ associated to the eigenvalues $\lambda_{n}=-\frac{n^{2} \pi^{2}}{a^{2}}$ with $\varphi_{n}(x)=\sqrt{\frac{2}{a}} \sin \left(\frac{n \pi x}{a}\right)$.

Each system $\left(S_{i}\right)$ is augmented with the output equation corresponding to a sensor $(D, f)$,

$$
\left(E_{i}\right) y_{i}(t)=\left\langle f, z_{i}(t)\right\rangle_{L^{2}(D)} ; 0<t<T
$$

According to proposition $8,(\Omega, g)$ dominates $(\Omega, h)$ with respect to the sensor $(\Omega, f)$, if and only if,

$$
\left[\forall n \in \mathbb{N}^{*},\left\langle g, \varphi_{n}\right\rangle\left\langle f, \varphi_{n}\right\rangle=0\right] \Rightarrow\left[\forall n \in \mathbb{N}^{*},\left\langle h, \varphi_{n}\right\rangle\left\langle f, \varphi_{n}\right\rangle=0\right]
$$

Let $m, n \in \mathbb{N}^{*}$ such that $m \neq n$. We suppose that $\left(S_{1}\right)$ and $\left(S_{2}\right)$ are respectively excited by the actuators $\left(\Omega, \varphi_{n}\right)$ and $\left(\Omega, \varphi_{m}\right)$., i.e. $g=\varphi_{n}$ and $h=\varphi_{m}$.

Then

- $(\Omega, g)$ dominates $(\Omega, h)$ with respect to the sensor $\left(\Omega, \varphi_{n}\right)$ and

- $(\Omega, h)$ dominates $(\Omega, g)$ with respect to the sensor $\left(\Omega, \varphi_{m}\right)$.
Let us also note that in the one dimension case, any operators $B_{1}$ and $B_{2}$ are comparable. this is not always possible in the two-dimension case which will be examined in the next section.

\subsection{Two Dimension Case}

Now, we consider the case where $\Omega=] 0,1[\times] 0,1[$ and 
the systems described by the following equations

$$
\begin{aligned}
& \left(S_{1}\right) \begin{cases}\left.\frac{\partial z_{1}(x, y, t)}{\partial t}=\Delta z_{1}(x, y, t)+g_{1}(x, y) u_{1}(t)+g_{2}(x, y) u_{2}(t) \text { in } \Omega \times\right] 0, T[ \\
z_{1}(x, y, t)=0 & \text { in } \Gamma \times] 0, T[ \\
z_{1}(x, y, 0)=0 & \text { in } \Omega\end{cases} \\
& \left(S_{2}\right) \begin{cases}\left.\frac{\partial z_{2}(x, y, t)}{\partial t}=\Delta z_{2}(x, y, t)+h_{1}(x, y) v_{1}(t)+h_{2}(x, y) v_{2}(t) \text { in } \Omega \times\right] 0, T[ \\
z_{2}(x, y, t)=0 & \text { in } \Gamma \times] 0, T[ \\
z_{2}(x, y, 0)=0 & \text { in } \Omega\end{cases}
\end{aligned}
$$

Here, we have $Z=L^{2}(\Omega)$ and $A z=\Delta z=\frac{\partial z}{\partial x^{2}}+\frac{\partial z}{\partial y^{2}}$, for $z \in D(A)=H^{2}(\Omega) \cap H_{0}^{1}(\Omega) . \quad A$ admits a complete orthonormal system of eigenfunctions $\left(\varphi_{m, n}\right)_{m, n \in \mathbb{N}^{*}}$ associated to the eigenvalues $\left(\lambda_{m, n}\right)_{m, n \in \mathbb{N}^{*}}$ defined by

$$
\left\{\begin{array}{l}
\lambda_{m, n}=-\left(m^{2}+n^{2}\right) \pi^{2} \\
\varphi_{m, n}(x, y)=2 \sin (m \pi x) \sin (n \pi y)
\end{array}\right.
$$

$\left(S_{1}\right)$ and $\left(S_{2}\right)$ are respectively augmented with the output equations

$$
\left.\left(E_{1}\right) y_{1}(t)=\left(\left\langle f_{1}, z_{1}(t)\right\rangle_{L^{2}\left(D_{1}\right)}\left\langle f_{2}, z_{1}(t)\right\rangle_{L^{2}\left(D_{2}\right)}\right),\right] 0, T[
$$

and

$$
\left.\left(E_{2}\right) y_{2}(t)=\left(\left\langle f_{1}, z_{2}(t)\right\rangle_{L^{2}\left(D_{1}\right)},\left\langle f_{2}, z_{2}(t)\right\rangle_{L^{2}\left(D_{2}\right)}\right),\right] 0, T[
$$

Let us first note that: $200=14^{2}+2^{2}=10^{2}+10^{2}$, then -200 is a double eigenvalue, corresponding to the eigenfunctions $\varphi_{10,10}$ and $\varphi_{2,14}$.

By the same, $250=15^{2}+5^{2}=13^{2}+9^{2}$, then -250 is also a double eigenvalue, corresponding to the eigenfunctions $\varphi_{5,15}$ and $\varphi_{9,13}$.

The examples given hereafter show the following situations :

- An actuator may dominates another one with respect to a sensor.

- None of the systems does not dominates the other.

Example 9. In the case where $g_{1}=\varphi_{10,10}, g_{2}=0$, $h_{1}=0, h_{2}=\varphi_{5,15}, f_{1}=\varphi_{10,10}$ and $f_{2}=\varphi_{2,14}$, we have

$$
\bigcap_{n \geq 1} \operatorname{ker}\left(M_{n} G_{n}^{t r}\right)=\mathbb{R}(0,1) \text { and } \bigcap_{n \geq 1} \operatorname{ker}\left(Q_{n} G_{n}^{t r}\right)=\{0\}
$$

where $\mathbb{R}(0,1)$ denotes the y-axis. Therefore $\left(S_{2}\right)$ dominates $\left(S_{1}\right)$ with respect to the corresponding out- put operator $C$.

On the other hand, for $g_{1}=\varphi_{10,10}, g_{2}=0, h_{1}=0$, $h_{2}=\varphi_{5,15}$ and $f_{1}=\varphi_{5,15}, f_{2}=\varphi_{9,13}$, we have

$$
\begin{aligned}
& \bigcap_{n \geq 1} \operatorname{ker}\left(M_{n} G_{n}^{t r}\right)=\{0\} \\
& \text { and } \bigcap_{n \geq 1} \operatorname{ker}\left(Q_{n} G_{n}^{t r}\right)=\mathbb{R}(1,0)
\end{aligned}
$$

where $\mathbb{R}(1,0)$ denotes the $x$-axis. Then $\left(S_{1}\right)$ dominates $\left(S_{2}\right)$ with respect to the corresponding output operator $C$.

Example 10. Now, for $g_{1}=\varphi_{10,10}, g_{2}=0, h_{1}=0$, $h_{2}=\varphi_{2,14}, f_{1}=\varphi_{10,10}$ and $f_{2}=\varphi_{2,14}$, we have

$$
\begin{aligned}
& \bigcap_{n \geq 1} \operatorname{ker}\left(M_{n} G_{n}^{t r}\right)=\mathbb{R}(0,1) \\
& \text { and } \bigcap_{n \geq 1} \operatorname{ker}\left(Q_{n} G_{n}^{t r}\right)=\mathbb{R}(1,0)
\end{aligned}
$$

Then none of the operators $B_{1}$ and $B_{2}$ does not dominates the other.

\section{Domination of Output Operators}

In this section, we introduce and we study the notion of domination for observed systems (output operators) with respect to an input one. We consider first a dual problem where the control concerns the initial state, and then a general controlled system.

\subsection{A Dual Problem}

In this section, we examine a dual problem concerning the output operators and observed systems. We consider the system

$$
\left\{\begin{array}{l}
\dot{z}(t)=A z(t) ; 0<t<T \\
z(0)=B u_{0}
\end{array}\right.
$$

The initial state $z_{0}$ depends on an input operator $B$ 
and is of the form $z(0)=B u_{0}$. We assume that $A$ is a linear operator with a domain $D(A)$ dense in $Z$, a separable Hilbert space, and generates a strongly continuous semi-group $(S(t))_{t \geq 0}$ on the state $Z$. $B \in$ $\mathcal{L}(U, Z), \quad u_{0} \in U ; \quad U$ is a Hilbert space. The system $(S)$ is augmented with the following output equations

$$
\begin{aligned}
& y_{1}(t)=C_{1} z(t) ; 0<t<T \\
& y_{2}(t)=C_{2} z(t) ; 0<t<T
\end{aligned}
$$

For $i=1,2$; the observations are given by

$$
y_{i}(t)=C_{i} S(t) B u_{0} ; 0<t<T
$$

We have $y_{i}()=.K_{i}(.) u_{0}$, with

$$
K_{i}=C_{i} S(.) B
$$

Its adjoint operator is defined by

$$
K_{i}^{*} y=\int_{0}^{T} B^{*} S^{*}(t) C_{i}^{*} y(t) \mathrm{d} t
$$

Noting $B_{i}=C_{i}^{*} ; \quad i=1,2 ; B=C^{*}$ and considering the dual systems

$$
\left(S_{i}^{*}\right)\left\{\begin{array}{l}
\dot{z}_{i}(t)=A^{*} z_{i}(t)+B_{i} u_{i}(t) ; 0<t<T \\
z_{i}(0)=z_{0} \\
y_{i}(t)=C z_{i}(t)
\end{array}\right.
$$

and

$$
\left(\tilde{S}_{i}\right)\left\{\begin{array}{l}
\dot{z}(t)=A z(t) ; 0<t<T \\
z(0)=B u_{0} \\
y_{i}(t)=C_{i} z(t)
\end{array}\right.
$$

we obtain the following characterization result.

Proposition 11. $\operatorname{Im}\left(K_{2}^{*}\right) \subset \operatorname{Im}\left(K_{1}^{*}\right)$ (respectively $\left.\overline{\operatorname{Im}\left(K_{2}^{*}\right)} \subset \overline{\operatorname{Im}\left(K_{1}^{*}\right)}\right)$ if and only if, the controlled system $\left(S_{1}^{*}\right)$ dominates $\left(S_{2}^{*}\right)$ exactly (respectively weakly).

From this general result, one can deduce analogous results and similar properties to those given in previous sections.

\subsection{Domination of Output Operators}

We consider the following linear distributed system

$$
\text { (S) }\left\{\begin{array}{l}
\dot{z}(t)=A z(t)+B u(t) ; 0<t<T \\
z(0)=z_{0}
\end{array}\right.
$$

where $A$ generates a s.c.s.g. $(S(t))_{t \geq 0}$ on the state space $Z ; B \in \mathcal{L}(U, Z)$ and $u \in L^{2}(0, T ; U) ; \quad U$ is the control space and the system (S) is augmented with the output equations

$$
y_{i}(t)=C_{i} z(t), 0<t<T ; i=1,2 .
$$

where $C_{i} \in L(Z, Y) ; \quad i=1,2 ; \quad Y$ is an Hilbert space.
The observation with respect to operator $C_{i}$ at the final time $T$, is given by

$$
y_{i}(T)=C_{i} S(T) z_{0}+C_{i} H u
$$

We introduce hereafter the appropriate notion of domination for the considered case.

Definition 12. We say that

1) $C_{1}$ dominates $C_{2}$ exactly with respect to the system (S) (or the pair $(A, B))$ on $[0, T]$, if $\operatorname{Im}\left(C_{2} H\right) \subset$ $\operatorname{Im}\left(C_{1} H\right)$.

2) $C_{1}$ dominates $C_{2}$ weakly with respect to the system (S) (or the pair $(A, B))$ on $[0, T]$, if $\overline{\operatorname{Im}\left(C_{2} H\right)} \subset$ $\overline{\operatorname{Im}\left(C_{1} H\right)}$.

Here also, we can deduce similar characterization results in the weak and exact cases. On the other hand, one can consider a natural question on a possible transitivity of such a domination. As it will be seen, this may be possible under convenient hypothesis. In order to examine this question, we consider without loss of generality, the linear distributed systems with the same dynamics $A$ $\left(A_{1}=A_{2}=A\right)$.

$$
\begin{aligned}
& \left(S_{1}\right)\left\{\begin{array}{l}
\dot{z}_{1}(t)=A z_{1}(t)+B_{1} u_{1}(t) ; 0<t<T \\
z_{1}(0)=z_{1,0}
\end{array}\right. \\
& \left(S_{2}\right)\left\{\begin{array}{l}
\dot{z}_{2}(t)=A z_{2}(t)+B_{2} u_{2}(t) ; 0<t<T \\
z_{2}(0)=z_{2,0}
\end{array}\right.
\end{aligned}
$$

where $A$ generates a s.c.s.g. $(S(t))_{t \geq 0}$ on the state space $Z ; B_{1} \in \mathcal{L}\left(U_{1}, Z\right), B_{2} \in \mathcal{L}\left(U_{2}, Z\right), u_{1} \in$ $L^{2}\left(0, T ; U_{1}\right), u_{2} \in L^{2}\left(0, T ; U_{2}\right) ; U_{1}$ and $U_{2}$ are two control spaces. The systems $\left(S_{1}\right)$ and $\left(S_{2}\right)$ are augmented with the output equations

$$
\begin{aligned}
& \left(E_{1, i}\right): y_{i, 1}(t)=C_{1} z_{i}(t) ; i=1,2 \\
& \left(E_{2, j}\right): y_{j, 2}(t)=C_{2} z_{j}(t) ; j=1,2
\end{aligned}
$$

where $C_{i} \in \mathcal{L}(Z, Y)$, for $i=1,2 ; Y$ is a Hilbert space. The observations with respect to operator $C_{1}$ at the final time $T$ are respectively given by

$$
\begin{aligned}
& y_{1,1}(T)=C_{1} S(T) z_{1,0}+C_{1} H_{1} u_{1} \\
& y_{2,1}(T)=C_{1} S(T) z_{2,0}+C_{1} H_{2} u_{2}
\end{aligned}
$$

By the same, the observations with respect to operator $C_{2}$ at time $T$ are given by

$$
\begin{aligned}
& y_{1,2}(T)=C_{2} S(T) z_{1,0}+C_{2} H_{1} u_{1} \\
& y_{2,2}(T)=C_{2} S(T) z_{2,0}+C_{2} H_{2} u_{2}
\end{aligned}
$$

We have the following result deriving from the definitions.

Proposition 13. If the following conditions are satisfied

1) $B_{1}$ dominates $B_{2}$ exactly (respectively weakly) 
with respect to operator $C_{1}$,

2) $C_{1}$ dominates $C_{2}$ exactly (respectively weakly) with respect to operator $B_{2}$,

3) $C_{2}$ dominates $C_{1}$ exactly (respectively weakly) with respect to operator $B_{1}$,

then $B_{1}$ dominates $B_{2}$ exactly (respectively weakly) with respect to operator $C_{2}$.

We examine hereafter, the relationship between the notions of domination and compensation.

\subsection{Domination and Compensation}

In this section, we study the relationship between the notions of domination and compensation [7,8]. We consider without loss of generality, the following systems.

$$
\begin{aligned}
& \left(\mathcal{S}_{1}\right)\left\{\begin{array}{l}
\dot{z}_{1}(t)=A z_{1}(t)+d_{1}(t)+B_{1} u_{1}(t), 0<t<T \\
z_{1}(0)=z_{1,0}
\end{array}\right. \\
& \left(\mathcal{S}_{2}\right)\left\{\begin{array}{l}
\dot{z}_{2}(t)=A z_{2}(t)+d_{2}(t)+B_{2} u_{2}(t), 0<t<T \\
z_{2}(0)=z_{2,0}
\end{array}\right.
\end{aligned}
$$

where $A$ generates a s.c.s.g. $(S(t))_{t>0}$ on the state space $Z ; B_{1} \in \mathcal{L}\left(U_{1}, Z\right), \quad B_{2} \in \mathcal{L}\left(U_{2}, Z\right), \quad u_{1} \in L^{2}\left(0, T ; U_{1}\right)$, $u_{2} \in L^{2}\left(0, T ; U_{2}\right), d_{1}$ and $d_{2} \in L^{2}(0, T ; Z) ; U_{1}$ and $U_{2}$ are two control spaces. $\left(S_{1}\right)$ and $\left(S_{2}\right)$ are respectively augmented with the output equations

$$
\begin{aligned}
& \left(\mathcal{E}_{1}\right) y_{1}(t)=C_{1} z_{1}(t) \\
& \left(\mathcal{E}_{2}\right) y_{2}(t)=C_{2} z_{2}(t)
\end{aligned}
$$

The states of these systems at the final time $T$ are respectively given by

$$
\begin{aligned}
& z_{1}(T)=S(T) z_{0}+H_{1} u_{1}+H d_{1} \\
& z_{2}(T)=S(T) z_{0}+H_{2} u_{2}+\tilde{H} d_{2}
\end{aligned}
$$

where the operators $H_{i} ; i=1,2$ and $\tilde{H}$ are defined by

$$
\begin{aligned}
& H_{i}: L^{2}\left(0, T ; U_{i}\right) \rightarrow Z \\
& u_{i} \rightarrow \int_{0}^{T} S(T-s) B_{i} u_{i}(s) \mathrm{d} s \\
& \tilde{H}: L^{2}(0, T ; Z) \rightarrow Z \\
& d \rightarrow \int_{0}^{T} S(T-s) d(s) \mathrm{d} s
\end{aligned}
$$

The corresponding observations are given by

$$
\begin{aligned}
& y_{1, f}(T)=C S(T) z_{0}+\mathrm{CH}_{1} u_{1}+C R d_{1} \\
& y_{2, f}(T)=C S(T) z_{0}+\mathrm{CH}_{2} u_{2}+C R d_{2}
\end{aligned}
$$

and $R f=\tilde{H} f$. First let us recall the notion of compensation.

Definition 14. The system $\left(\mathcal{S}_{i}\right)$ augmented with out- put equation $\left(\mathcal{E}_{i}\right)\left(\right.$ or $\left.\left(\mathcal{S}_{i}\right)+\left(\mathcal{E}_{i}\right)\right)$ is

1) exactly remediable on $[0, T]$ if for any $d_{i} \in L^{2}(0, T ; Z)$, there exists $u_{i} \in L^{2}\left(0, T ; U_{i}\right)$ such that $C_{i} H_{i} u_{i}+R d_{i}=0$, or equivalently

$$
\operatorname{Im}\left(C_{i} R\right) \subset \operatorname{Im}\left(C_{i} H_{i}\right)
$$

2) weakly remediable on $[0, T]$ if for any $d_{i} \in$ $L^{2}(0, T ; Z)$ and any $\epsilon>0$, there exists $u_{i} \in L^{2}\left(0, T ; U_{i}\right)$ such that $\left\|C_{i} H_{i} u_{i}+R d_{i}\right\|<\epsilon$, or equivalently

$$
\operatorname{Im}\left(C_{i} R\right) \subset \overline{\operatorname{Im}\left(C_{i} H_{i}\right)}
$$

Here, the question is not to examine if a system is (or not) remediable (for this one can see $[7,8]$ ), but to study the nature of the relation between the notions of domination and compensation, respectively in the exact and weak cases. We have the following result.

Proposition 15. If the following conditions are verified 1) $\left(\mathcal{S}_{1}\right)+\left(\mathcal{E}_{1}\right)$ is exactly (respectively weakly) remediable.

2) $C_{2}$ dominates $C_{1}$ exactly (respectively weakly) with respect to the operator $B_{1}$.

3) $\operatorname{Im}\left(C_{2} R\right) \subset \operatorname{Im}\left(C_{1} R\right) \quad$ (respectively $\overline{\operatorname{Im}\left(C_{2} R\right)} \subset$ $\left.\overline{\operatorname{Im}\left(C_{1} R\right)}\right)$.

then $\left(\mathcal{S}_{1}\right)+\left(\mathcal{S}_{2}\right)$ is exactly (respectively weakly) remediable.

We have the similar result concerning the output domination and the remediability notion.

Proposition 16. If the following conditions are satisfied

1) $\left(\mathcal{S}_{1}\right)+\left(\mathcal{E}_{1}\right)$ is exactly (respectively weakly) remediable.

2) $B_{2}$ dominates $B_{1}$ exactly (respectively weakly) with respect to the operator $C_{1}$.

then $\left(\mathcal{S}_{2}\right)+\left(\mathcal{E}_{1}\right)$ is exactly (respectively weakly) remediable.

Let us note that this section is a generalization of the previous one where $d(t)$ has the form $B_{2} u_{2}(t)$. The results can be applied easily to a diffusion system and to other systems and situations.

\section{REFERENCES}

[1] L. Afifi, A. El Jai and E. M. Magri, "Domination and Compensation in Finite Dimension Dynamical Systems," Applied Mathematical Sciences, Vol. 4, No. 49, 2008, pp. 2443-2457.

[2] L. Afifi, A. El Jai and E. M. Magri, "Weak and Exact Domination in Distributed Systems," International Journal of Applied Mathematics and Computer Science, Vol. 20, No. 3, 2010, pp. 419-426. doi:10.2478/v10006-010-0031-5

[3] L. Afifi, N. Amimi, A. El Jai and E. M. Magri, "Regional Domination in Distributed Systems," Applied Mathematical Sciences, Vol. 6, No. 19, 2012, pp. 913-924. 
[4] A. El Jai and A. J. Pritchard, "Sensors and Actuators in Distributed Systems Analysis," Ellis Horwood Series in Applied Mathematics, J. Wiley, 1987.

[5] R. F. Curtain and A. J. Pritchard, "Infinite Dimensional Linear Systems Theory," Lecture Notes in Control and Information Sciences, Vol. 8, 1978. doi:10.1007/BFb0006761

[6] R. F. Curtain and H. J. Zwart, "An Introduction to Infinite-Dimensional Linear Systems Theory," Texts in Applied Mathematics, Springer-Verlag, New York, 1995. doi:10.1007/978-1-4612-4224-6

[7] L. Afifi, A. Chafiai and A. El Jai, "Regionally Efficient and Strategic Actuators," International Journal of Systems Science, Vol. 33, No. 1, 2002, pp. 1-12. doi:10.1080/002077202317216884

[8] L. Afifi, A. El Jai and E. Zerrik, "Systems Theory: Regional Analysis of Infinite-Dimensional Linear Systems," Presses Universitaires de Perpignan, 2012.

[9] A. V. Balakrishnan, “Applied Functional Analysis," Springer Verlag, 1981.

[10] L. Berrahmoune, "Localisation D'actionneurs pour la Contrôlabilité de Systèmes Paraboliques et Hyperboliques," Application par Dualité à la Localisation de Capteurs, Thèse de 3ème Cycle, Université Mohammed V, Rabat, 1984.

[11] J. L. Lions, "Contrôlabilité Exacte, Perturbations et Stabilisation des Systèmes Distributes," Masson, Paris, 1988. 